\title{
Bessel beams and Gaussian beams as information carriers in free space optical interconnects systems: a comparison study
}

\author{
Nedal Al-Ababneh \\ Department of Electrical Engineering, Jordan University of Science and Technology, Jordan
}

\begin{tabular}{|c|c|}
\hline Article Info & ABSTRACT \\
\hline Article history: & We introduce models for crosstalk estimation in a lens-based free space \\
\hline Received Feb 10, 2019 & $\begin{array}{l}\text { optical interconnects that use non-diffracting Bessel beams and Gaussian } \\
\text { beams. We perform a comparison study for the performance two systems. }\end{array}$ \\
\hline Revised Apr 11, 2019 & The optical field at the detector plane was derived for the two beam profiles. \\
\hline Accepted Apr 20, 2019 & $\begin{array}{l}\text { In both cases the expressions for the output optical filed are expressed in } \\
\text { terms of complex Gaussian functions. The performance of the system for the }\end{array}$ \\
\hline $\begin{array}{l}\text { Keywords: } \\
\text { Free space optical interconnects }\end{array}$ & $\begin{array}{l}\text { two beams is evaluated and compared. Using simulation results we show that } \\
\text { the use of Bessel beam gives superior results to that of using Gaussian beam } \\
\text { for large interconnects distance. }\end{array}$ \\
\hline
\end{tabular}

Diffraction crosstalk

Gaussian beams

Non-diffracting Bessel beams

Copyright () 2019 Institute of Advanced Engineering and Science. All rights reserved.

Corresponding Author:

Nedal Al-Ababneh,

Department of Electrical Engineering,

Jordan University of Science \& Technology,

Irbid, Jordan.

Email: nedalk@ just. edu.jo

\section{INTRODUCTION}

Light propagation diffraction is considered as one of the important problems that affects the performance of free space optical interconnects (FSOIs) systems, namely, the signal-to-crosstalk ratio [1-5]. In these FSOIs systems light sources with Gaussian profiles are usually used. The crosstalk in these Gaussian-based systems is prominent and careful design is required to reduce its effect [6-9]. One interesting method to reduce the diffraction, particularly in board-to board applications with large interconnect distance, is the use of non-diffracting Bessel beams [10-12]. In [10] an efficient conversion of light from a laser diode into a Bessel beam whose axial intensity varies almost uniformly with distance using only a holographic optical element is explained. An interesting shadowing of the Bessel beam is demonstrated where on blocking the intense central spot the propagating ring pattern acts to reform the central spot a short distance after the obstruction. This behavior together with the long propagation range for the Bessel beam's central spot are considered for multi-board optical interconnects.

In [11] authors present high-density pseudo-non-diffracting beam arrays produced by using holographic method. The demonstrated pseudo-non-diffracting beams can keep their central spot size of about $95 \mu \mathrm{m}$ within a distance of $40 \mathrm{~cm}$. The center-to-center beam separation is $250 \mu \mathrm{m}$, which is much smaller than those achieved by collimated Gaussian beams with the same interconnect range. The small central lobe sizes can further avoid the use of collection/focusing lenses at the high-speed photodetector receivers. The concept of using non-diffracting beam in a lensless free space optical interconnects was investigated in [12]. It was shown that the performance of the FSOIs system using non-diffracting beams outperforms that which uses Gaussian beams especially for large interconnects distance. However, it was explained that the side lobes of the Bessel beams overlap with the neighbor channels and increase the crosstalk. Most of the work presented in the literature regarding the use of nondiffrating Bessel beams 
was based on some experimental demonstration. In fact, modelling the crosstalk in these systems is necessary for the design and for the sake of finding solutions and methods to reduce crosstalk.

In this article, we introduce models for crosstalk estimation for the optical interconnect system that use non-diffracting Bessel beams in a lens-based free space optical interconnects. Closed form expressions for the light intensity distribution at the detector plane are derived for Gaussian and Bessel beams the by expanding the micro-lens aperture in term complex Gaussian functions. Using these expressions, the signal to crosstalk ratio is evaluated and used as a performance measure. A comparison study is performed between the system that uses the Bessel beam and the one that uses the Gaussian beams.

The main goals of this paper is to (1) introduce crosstalk model for nondifffacting beam based free space optical interconnects systems (2) compare the interconnect performance using these beams with that of Gaussian beam based systems (3) Recommend one of these systems for a given application.

\section{OUTPUT OPTICAL FIELD USING DIFFRACTION INTEGRAL}

The lens system considered in this paper is shown in Figure 1. This system contains VCSELs array, lens array, and detectors array located at the output plane.

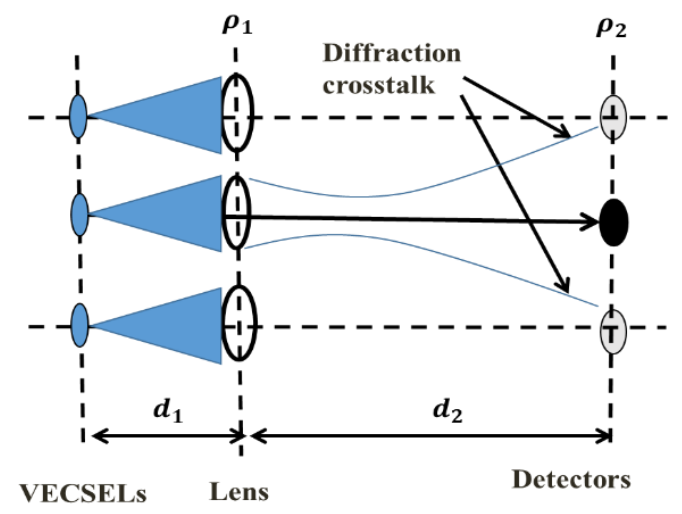

Figure 1. Micro-lens based FSOIs

The input plane is the plane $\rho=\rho_{1}$ and the output plane is the plane $\rho=\rho_{2}$. The distance between these two planes is $d_{2}$. The light sources (VCSELs) array is placed at the front focal length of the lens. For an input optical field $E_{1}\left(\rho_{1}, \theta_{1}\right)$, the output optical field $\mathrm{E}_{2}\left(\rho_{2}, \theta_{2}\right)$ can be found using Collins diffraction integral as follows [13].

$$
\begin{aligned}
\mathrm{E}_{2}\left(\rho_{2}, \theta_{2}\right) & =\frac{i k}{2 \pi b} \int_{0}^{\infty} \int_{0}^{2 \pi} \mathrm{E}_{1}\left(\rho_{1}, \theta_{1}\right) A\left(\rho_{1}\right) \\
& \times \exp \left[\begin{array}{c}
-\frac{i k a}{2 b} \rho_{1}^{2}-\frac{i d k}{2 b} \rho_{2}^{2} \\
+\frac{i k \rho_{1} \rho_{2}}{b} \cos \left(\theta_{1}-\theta_{2}\right)
\end{array}\right] \rho_{1} d \rho_{1} d \theta_{1}
\end{aligned}
$$

$\rho_{1}, \theta_{1}$ and $\rho_{2}, \theta_{2}$ are the cylindrical coordinates. $A\left(\rho_{1}\right)$ is the lens' aperture function. $k=2 \pi / \lambda$ is the wave number, and $\lambda$ is the wavelength. $a, b, c$, and $d$ are the transfer matrix elements of the FSOIs system and are given by:

$$
\left[\begin{array}{ll}
a & b \\
c & d
\end{array}\right]=\left[\begin{array}{ll}
1-\left(\frac{d_{2}}{d_{1}}\right) & d_{2} \\
\frac{-1}{d_{1}} & 1
\end{array}\right]
$$




\section{OUTPUT OPTICAL FIELD FOR GAUSSIAN INPUT BEAM}

Assuming Gaussian model for the input optical field, $E_{1}\left(\rho_{1}, \theta_{1}\right)$ can be written as

$$
\mathrm{E}_{1}\left(\rho_{1}, \theta_{1}\right)=\exp \left[-\frac{\rho_{1}^{2}}{\omega_{1}^{2}}\right]
$$

$\omega_{1}$ the beam radius at the front surface of the lens and is given by

$$
\omega_{1}=\omega_{0} \sqrt{1+\frac{\lambda^{2} d_{1}^{2}}{\pi^{2} \omega_{0}^{4}}}
$$

$\omega_{0}$ is the waist radius of the VCSEL's beam. The integral in (1) can be evaluated by using (3) and recalling integral formula

$$
J_{0}(x)=\frac{1}{2 \pi} \int_{0}^{2 \pi} \exp (i x \cos \theta) d \theta
$$

to obtain

$$
E_{2}\left(\rho_{2}\right)=\frac{i k}{b} \exp \left(-\frac{i k d \rho_{2}^{2}}{2 b}\right) \int_{0}^{\infty} \exp \left(-\rho_{1}^{2} / q^{2}\right) J_{0}\left(\frac{k \rho_{2}}{b} \rho_{1}\right) A\left(\rho_{1}\right) \rho_{1} d \rho_{1}
$$

For a circular lens with $a_{1}$ radius, the aperture function is given by

$$
A\left(\rho_{1}\right)= \begin{cases}1 & \rho_{1} \leq a_{1} \\ 0 & \rho_{1} \succ a_{1}\end{cases}
$$

Substituting in (7) for $A\left(\rho_{1}\right)$ from (8), to obtain

$$
\begin{aligned}
& E_{2}\left(\rho_{2}\right)=\frac{i k}{b} \exp \left(-\frac{i k d \rho_{2}^{2}}{2 b}\right) \int_{0}^{a_{1}} \exp \left(-\rho_{1}^{2} / q^{2}\right) J_{0}\left(\alpha \rho_{1}\right) \rho_{1} d \rho_{1} \\
& \frac{1}{q^{2}}=\frac{1}{\omega_{1}^{2}}+\frac{i k a}{2 b} \\
& \alpha=\frac{k \rho_{2}}{b}
\end{aligned}
$$

Equation (8) is a formula for the output optical field for a Gaussian beam propagating through the lens-based FSOIs system shown in Figure 1. The output optical field can be evaluated in a closed form by expanding the lens' aperture function in terms of complex Gaussian functions $[14,15]$ as follows

$$
A\left(\rho_{1}\right)=\sum_{n=1}^{N} A_{n} \exp \left(-\frac{B_{n}}{a_{1}^{2}} \rho_{1}^{2}\right)
$$

where $A_{n}$ and $B_{n}$ are the expansion and Gaussian complex coefficients, respectively. The values of these coefficients can be found by optimization-computation directly [14]. On substituting in (8) for $A\left(\rho_{1}\right)$ 
from (11), to get

$$
\begin{aligned}
& E_{2}\left(\rho_{2}\right)=\frac{i k}{b} \exp \left(-\frac{i k d \rho_{2}^{2}}{2 b}\right) \sum_{n=1}^{N} A_{n} \int_{0}^{\infty} \exp \left(-p \rho_{1}^{2}\right) J_{0}\left(\alpha \rho_{1}\right) \rho_{1} d \rho_{1} \\
& p=\frac{1}{q^{2}}+\frac{B_{n}}{a_{1}^{2}}=\frac{1}{\omega_{1}^{2}}+\frac{i k a}{2 b}+\frac{B_{n}}{a_{1}^{2}}
\end{aligned}
$$

The integral in (12) can be solved by recalling the integral formula

$$
\int_{0}^{\infty} \exp (-p x) J_{0}(2 \sqrt{x} \sqrt{\alpha}) d x=p^{-1} \exp \left(-\frac{\alpha}{p}\right)
$$

Using the integral in (14), the output optical filed in equation (12) can be expressed as

$$
\begin{aligned}
E_{2}\left(\rho_{2}\right) & =\frac{i k}{b} \exp \left(-\frac{i k d \rho_{2}^{2}}{2 b}\right) \sum_{n=1}^{N} A_{n} \int_{0}^{\infty} \exp \left(-p \rho_{1}^{2}\right) J_{0}\left(\alpha \rho_{1}\right) \rho_{1} d \rho_{1} \\
& =\frac{i k}{b} \exp \left(-\frac{i k d \rho_{2}^{2}}{2 b}\right) \sum_{n=1}^{N} \frac{A_{n}}{2 p} \exp \left(-\frac{k^{2}}{4 p b^{2}} \rho_{2}^{2}\right)
\end{aligned}
$$

Equation (15) is an approximate closed form solution for the diffraction integral of equation (8). Note that the solution is written in terms of the elements of the optical system transfer matrix.

\section{OUTPUT OPTICAL FIELD FOR BESSEL INPUT BEAM}

Assuming a Bessel non-diffracting beam for the input optical filed, $\mathrm{E}_{1}\left(\rho_{1}, \theta_{1}\right)$ at the front surface of the microlens can be given by

$$
\mathrm{E}_{1}\left(\rho_{1}, \theta_{1}\right)=\mathbf{J}_{0}\left(\sigma \rho_{1}\right)
$$

$\sigma$ is the the transverse wave number which controls the sharpness of the beam. Now, by substituting (16) into (1) and using (11) for the aperture and using the following two integrals

$$
\begin{aligned}
& \frac{1}{2 \pi} \int_{0}^{2 \pi} \exp \left[i x \cos \left(\theta_{2}-\theta_{1}\right)\right] \exp \left[-i n \theta_{1}\right] d \theta_{1} \\
& =i^{n} J_{n}(x) \exp \left[-i n \theta_{2}\right] \\
& \int_{0}^{\infty} \exp \left[-\gamma \rho^{2}\right] J_{n}(\alpha \rho) J_{n}(\beta \rho) \rho d \rho \\
& =\frac{(-1)^{n}}{2 \gamma} \times \exp \left[-\frac{i n \pi}{2}\right] \exp \left[-\frac{1}{4 \gamma}\left(\alpha^{2}+\beta^{2}\right)\right] J_{n}\left(\frac{i \alpha \beta}{2 \gamma}\right)
\end{aligned}
$$

The optical field at the detectors array can be found as

$$
\begin{aligned}
& \mathrm{E}_{2}\left(\rho_{2}, \theta_{2}\right)=\sum_{n=1}^{N} A_{n} \frac{k(-1)^{n}(\mathrm{i})^{\mathrm{n}+1}}{b q} \exp \left[-i m \theta_{2}+\frac{i n \pi}{2}\right] \\
& \exp \left[-\frac{i k}{b} d \rho_{2}^{2}\right] \exp \left[-\frac{1}{2 q}\left(\sigma^{2}+\frac{k^{2} \rho_{2}^{2}}{b^{2}}\right)\right] J_{n}\left(i \sigma \frac{k \rho_{2}}{q b}\right) \\
& q=\frac{2 B_{n}}{a_{1}^{2}}+\frac{i k a}{b}
\end{aligned}
$$




\section{CROSSTALK CALCULATIONS}

The crosstalk power received by the intended detector can be evaluated as the total power received by all other detectors (excluding the intended detector) from the light passing through the intended lens with only the intended source is on. Taking into account only the eight nearest detectors, the crosstalk power can be expressed as

$$
\begin{aligned}
P_{c}= & 4 \iint_{A_{1}}\left|\mathrm{E}_{2}\left(\rho_{2}, \theta_{2}\right)\right|^{2} \rho_{2} d \rho_{2} d \theta_{2} \\
& +4 \iint_{A_{2}}\left|\mathrm{E}_{2}\left(\rho_{2}, \theta_{2}\right)\right|^{2} \rho_{2} d \rho_{2} d \theta_{2}
\end{aligned}
$$

$A_{1}$ is the area of one of the four neighbor detectors and $A_{2}$ is the area of one of the next four neighbor detectors. The signal power is the power received by the intended detector assuming only the intended source is on and is given by:

$$
P_{s}=\iint_{A}\left|\mathrm{E}_{2}\left(\rho_{2}, \theta_{2}\right)\right|^{2} \rho_{2} d \rho_{2} d \theta_{2}
$$

$A$ is the area of the intended detector. Having determined the signal and the crosstalk power, the signal to crosstalk ratio, SCR, can be evaluated as

$$
S C R=\frac{P_{s}}{P_{c}}
$$

\section{NUMERICAL SIMULATIONS}

In this section we show numerical results to explain the advantages of using the Bessel beam as information carrier in free space optical interconnects. We also include numerical results for the optical system that uses the Gaussian beam as information carrier. In our simulations a wavelength of $\lambda=0.850 \mu \mathrm{m}$ is assumed for both beams. For the Bessel beam the transverse wave number $\sigma=2.5(\mu \mathrm{m})^{-1}$ and a beam radius of $5 \mu \mathrm{m}$ at the front surface of the lens for the Gaussian beam. The lens has a focal length and a diameter of 720 $\mu \mathrm{m}$ and $300 \mu \mathrm{m}$, respectively. The distance between neighboring detectors as well as neighboring light sources is $300 \mu \mathrm{m}$. Figure 2 shows the 1-D transverse profiles for the input field intensity for both the Bessel and Gaussian beams. The parameters of the beams are chosen to have almost the same width at the input plane. To show the benefits of using Bessel beam, the SCR versus the detector radius is plotted in Figure 3, 4 and 5 for three different values of interconnects distance. Comparing the three figures, we can see that the SCR for the FSOIs system using Bessel beam outperforms that of the system which uses the Gaussian beam for large interconnects length. In Figure 3 the interconnects length is small and using the Gaussian beam is still preferred since the diffraction can be tolerated. However, as the interconnects length increases the effect of the diffraction is apparent as shown in Figure 4 and Figure 5 and in this case the Bessel beam is preferred.

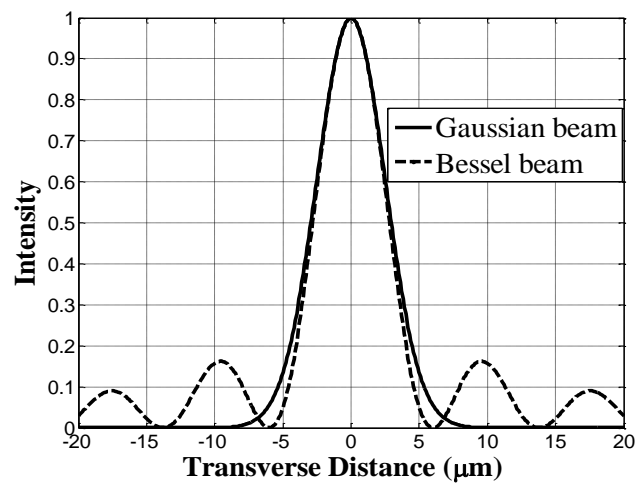

Figure 2. Input optical Field intensity for the two

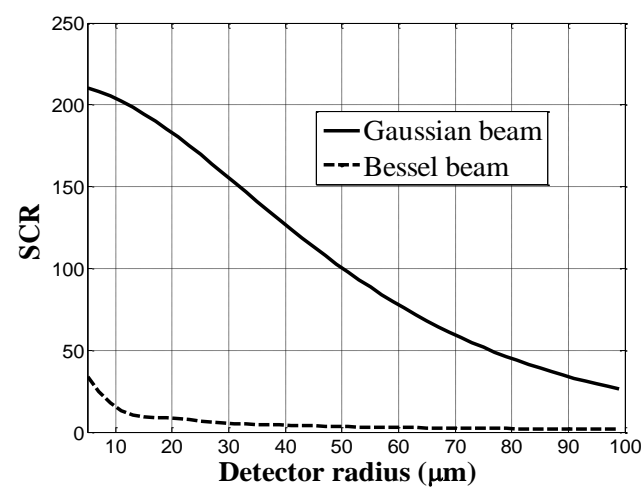

Figure 3. SCR versus detector radius with 


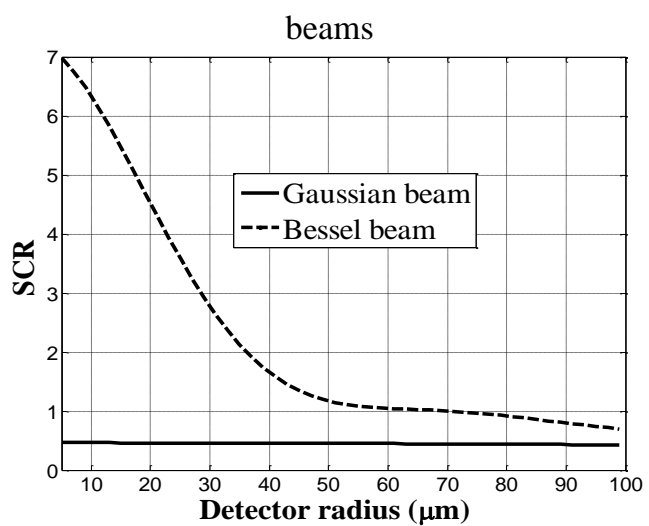

Figure 4. SCR versus detector radius with interconnect length of $8 \mathrm{~mm}$

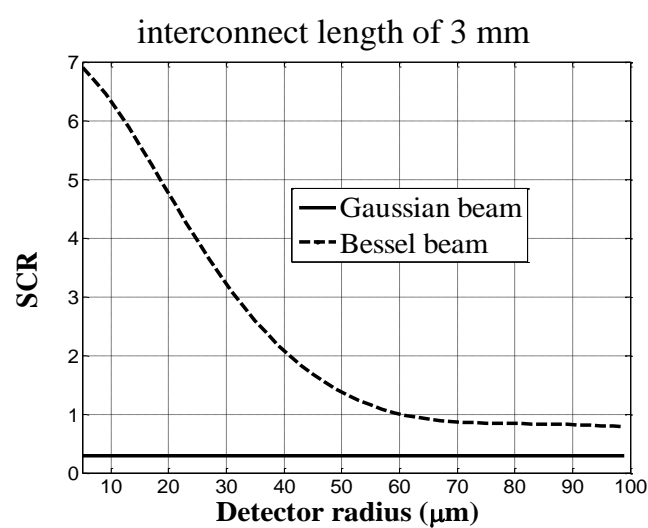

Figure 5. SCR versus detector radius with interconnect length of $10 \mathrm{~mm}$

\section{CONCLUSION}

Crosstalk models for the free space optical interconnects system have been introduced for two systems. The performance of two systems was analyzed using paraxial approximation. One of the optical systems used the Gaussian beam as information carrier and the other system used Bessel beams. Analytical formulas for output optical field at the detector plane were derived and used to evaluate the crosstalk which we used as the performance measure. The simulation results have shown that the use of Bessel beam gave superior results to that of using Gaussian beam at large interconnects lengths. For board-to board interconnects the use of nondiffracting beams is more reliable.

\section{REFERENCES}

[1] K. Wang, et al., "80 Gb/s Free-Space Reconfigurable Optical Interconnects with Carrierless-Amplitude-Phase Modulation and Space-Time Block Code," Optical Fiber Communication Conference. Optical Society of America, 2018.

[2] S. V. Winkle, et al., "Extending the Power-Efficiency and Performance of Photonic Interconnects for Heterogeneous Multicores with Machine Learning," in High Performance Computer Architecture (HPCA), 2018 IEEE International Symposium on, pp. 480-491, 2018.

[3] J. M. Kahn and D. A. B. Miller, "Communications expands its space," Nature photonics, vol/issue: 11(1), pp. 5, 2017.

[4] M. Châteauneuf, et al., "512-channel vertical-cavity surface-emitting laser based free-space optical link," Applied optics, vol/issue: 41(26), pp. 5552-5561, 2002.

[5] G. Kim, et al., "Crosstalk and interconnection distance considerations for board-to-board optical interconnects using 2-D VCSEL and microlens array," IEEE Photonics Technology Letters, vol/issue: 12(6), pp. 743-745, 2000.

[6] W. Hu, et al., "Crosstalk analysis of aligned and misaligned free-space optical interconnect systems," J. Opt. Soc. Am. A, vol/issue: 27(2), pp. 200-205, 2010.

[7] N. S. Petrovic and A. D. Rakic, "Modeling diffraction and imaging of laser beams by the mode-expansion method," J. Opt. Soc. Am. B, vol/issue: 22(3), pp. 556-566, 2005.

[8] F. F. Tsai, et al., "Analysis of optical channel cross talk for free-space optical interconnects in the presence of higher-order transverse modes," Appl. Opt., vol/issue: 44(30), pp. 6380-6387, 2005.

[9] S. Tang, et al., "Design limitations of highly parallel free-space optical interconnects based on array of vertical cavity surface-emitting laser diodes, microlenses, and photo detectors," J. Lightwave Technol., vol/issue: 12(11), pp. 1971-1975, 1994.

[10] R. P. MacDonald, et al., "Interboard optical data distribution by Bessel beam shadowing," Optics Communications, vol/issue: 122(4-6), pp. 169-177, 1996.

[11] C. Yu, et al., "High-density non-diffracting beam array for optical interconnection," Optics Communications, vol/issue: 177(1-6), pp. 369-376, 2000.

[12] N. Al-Ababneh and M. Testorf, "Analysis of free space optical interconnects based on non- diffracting beams," Optics Communications, vol. 242, pp. 393-400, 2004.

[13] S. A. Collins, "Lens-systems diffraction integral written in terms of matrix optics," J. Opt. Soc. Am., vol/issue: 60(9), pp. 1168-1177, 1970.

[14] J. J. Wen and M. A. Breazeale, "A diffraction beam field expressed as the superposition of Gaussian beams," J. Acoust. Soc. Am., vol/issue: 83(5), pp. 1752-1756, 1988.

[15] D. Ding and Y. Zhang, "Notes on the Gaussian beam expansion," J. Acoust. Soc. Am., vol/issue: 116(3), pp. 14011405, 2004. 


\section{BIOGRAPHIES OF AUTHORS}

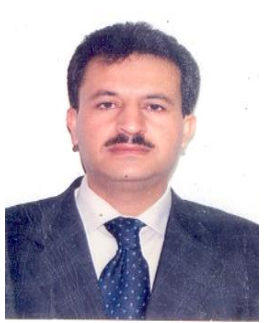

Nedal Al-Ababneh received his B.Sc. and M.Sc. degrees in Electrical Engineering from Jordan University of Science and Technology in 1993 and 1996 respectively. He received his Doctoral of Engineering degree in Electrical engineering from University of Massachusetts-Lowell (USA) in 2004. He is currently full professor with the department of electrical engineering in Jordan University of Science and Technology. His research interests are in free space optical interconnects and distributed detection systems. 